\title{
Magnetization reversal in spin patterns with complex geometry
}

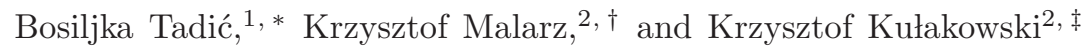 \\ ${ }^{1}$ Department for Theoretical Physics, Jozef Stefan Institute, P.O.Box 3000, SI-1001, Ljubljana, Slovenia. \\ ${ }^{2}$ Faculty of Physics and Applied Computer Science, AGH University of Science and Technology, \\ al. Mickiewicza 30, PL-30059 Kraków, Poland.
}

(Dated: November 16, 2018)

\begin{abstract}
We study field-driven dynamics of spins with antiferromagnetic interaction along the links of a complex substrate geometry, which is modeled by graphs of a controlled connectivity distribution. The magnetization reversal occurs in avalanches of spin flips, which are pinned by the topological constraints of the underlying graph. The hysteresis loop and avalanche sizes are analyzed and classified in terms of graph's connectivity and clustering. The results are relevant for magnets with a hierarchical spatial inhomogeneity and for design of nanoscale magnetic devices.

PACS numbers: 89.75.Da,75.60.Ej, 75.75.+a,
\end{abstract}

Introduction. Reversal processes are of great importance for technological applications, e.g., in information processing and memory devices. Often hysteresis curves with particular properties are required. In this respect novel "artificial solids", arrays of nanoscale magnetic particles [1], quantum cellular automata 2] and integrated functional nanosystems [3] offer challenging possibilities, yet to be understood. Currently methods are being developed for patterning, defining and measurements of the magnetic properties at nanometer scale [4]. Much less attention heve been devoted to the theoretical study of these systems [5].

In classical disordered ferromagnets 6], ferroelectrics [7] and systems with structural transformations [8] the reversal processes are accompanied by avalanches, which are directly related with the motion and pinning of the domain walls. The distribution of avalanches and the time-series of the magnetization bursts (generalized Barkhausen noise) exhibits universal scaling features, which can be used for the diagnostics of the underlying domain structure. The pinning of the domain walls is visualized as an effect of random disorder on a perfect crystal lattice, and commonly modeled by quenched random fields [9]. Despite of large theoretical efforts [9], the exact role of disorder in the emergent universality of noise has not yet been fully understood. In general, the stronger pinning implies smaller avalanches and reduced coercive fields (slimmer hysteresis loops). In the exact solution 10] in the case of Bethe lattice with large coordination number and weak disorder, a finite jump in the magnetization persists when the system size $N \rightarrow \infty$, suggesting true criticality (absence of a cut-off) in the avalanche distribution integrated along the hysteresis loop.

Variety of domain forms may be nucleated in ferroelectrics with low symmetry and long-range elastic forces 11]. On the other hand, the patterned nanoscale magnetic structures can be assembled in various geometries unrestricted by a crystal symmetry [3]. The magnetostatic interactions between neighbour nanoparticles and their shape anisotropy allow both ferromagnetic and an- tiferromagnetic coupling within nanoarrays [12]. The full impact of the assembly processes and the emergent geometries on physical properties of the integrated nanoscale devices calls for theoretical analysis.

The stochastic processes on complex networks attracted much attention recently [13]. In general, the properties of the process depend on the complexity of the underlying network. Compared to more familiar Bravais' lattices, the network geometry makes severe constraints to the dynamics by restricting the interaction pathways along the inhomogeneous structure of links. The network inhomogeneity proves as beneficial in the case of transport processes 14. The spin dynamics on networks has been less studied [15, 16].

In this Letter we study dynamics of spins attached to nodes of a complex network and slowly driven by ramping of the uniform external field. We assume antiferromagnetic interactions between spins along the links and observe avalanches of spin flips due to topological inhomogeneity of the network. An avalanche represents a fraction of spins that are reversed at current field value in order to minimize the energy within locally available geometry. We find numerically evidence of the hysteresis loop criticality which can be explained in terms of the structural properties of the network.

Structure. Our model of two-state spins attached to nodes of a complex network can be regarded as a model of an integrated nanosystem with a nontrivial architecture. The structure is grown by systematic addition and linking of nodes 15. At each growth step $i$ a node is added and linked to $M$ nodes selected among $i-1$ preexisting nodes. Selection of a target node, $k$, is given by a specified probability $p(k, i)$. For $p(k, i)=1 /(i-1)$, i.e., independent of properties of target nodes, the emergent network is known to have an exponential degree distribution. In contrast, the preferential linking, where the selection probability $p(k, i)$ depends on number of already acquired links $q(k, i)$, are shown to lead to a scale-free degree distribution 15, 17, 18]. Here we use the probability $p(k, i)=[1+q(k, i) / M] / 2 i$ to grow scale-free networks for 
the purpose of this work. We restrict our discussion to the simplest complex networks for the following reasons: (i) Their structure is well understood [15, 17, 18], in particular, there are no hidden topological properties and link correlations 18); (ii) Varying a single structural parameter $M$ we tune the graph clustering property. Both scale-free network (SFN) and exponential network (EXN) have a tree-like structure when $M=1$ (an example is shown in Fig. 11). For $M>1$ cycles (disregarding the direction of links) can appear, which strongly depend on the linking rule. For instance, for $M=5$, we find the average clustering coefficient [17] as 0.2803 in SFN, and 0.0553 in EXN for network size of $N=10^{3}$ nodes.

The network sparseness and structural inhomogeneity are the feature which can affect the spin dynamics. In the two network types introduced above, the profiles of the local connectivity (number of links attached to a node) are shown in the inset to Fig. 2] where $i$ denotes the order of addition of the node to the network. In the case of SFN of size $N$ the profile is given by the power-law dependence $\langle q(i, N)\rangle \propto(N / i)^{\gamma}$, with $\gamma=1 / 2$ related to the emergent power-law degree distribution $P(q) \propto q^{-3}$ ( see [15, 18] for more general scaling relations). In the case of EXN we have $\langle q(i, N)\rangle \propto \log (N / i)$. When $M>1$ the number of the elementary triangles or clustering $\langle\Delta(i)\rangle$ at a node $i$ (i.e., number of direct links among nearst-neighbors of $i$ ) also varies through the network. The clustering profiles for $M=5$ in SFN and EXN, exhibiting power-law tails, are also shown in the inset to Fig. 2

Spin Dynamics. A spin, $S= \pm 1$, is associated with each node on the network. The spins interact along the links connecting neighbouring nodes. We assume uniform anti-ferromagnetic interaction $J=-1$ and uniform external field $H$ according to the Hamiltonian

$$
\mathcal{H} \equiv-\sum_{i} h_{i} S_{i}=-\sum_{i, j>i} J C_{i j} S_{i} S_{j}-H \sum_{i} S_{i}
$$

The sum over pairs of nodes is restricted to the locally available links, which are given by the positive elements $C_{i j}$ of the adjacency matrix $\mathbf{C}$ of the graph. The interaction along these links is symmetrical. Therefore, the local field $h_{i}$, which is defined in Eq. (11), varies with the local connectivity profile $\langle q(i, N)\rangle$ of the graph.

We start from a negative saturation state in the field $H=-H_{\max }$, where $H_{\max }=q_{\max }+\delta$ is determined by the largest connectivity $q_{\max }$ (at hub node) for each network realization, and $\delta$ is a small shift which prevents $h_{i}=0$. Slowly increasing external field is outbalanced at some node where the interaction with other spins is the strongest. Flipping the spin at that node may launch a cascade of flips along the connections of the graph. The avalanche of flips is stopped when the energy is minimized at current field value. Then the field is increased again. This produces the step-wise increase of the magnetization with time and with field, as shown in Fig. 3] in a manner known as Barkhausen effect in systems with

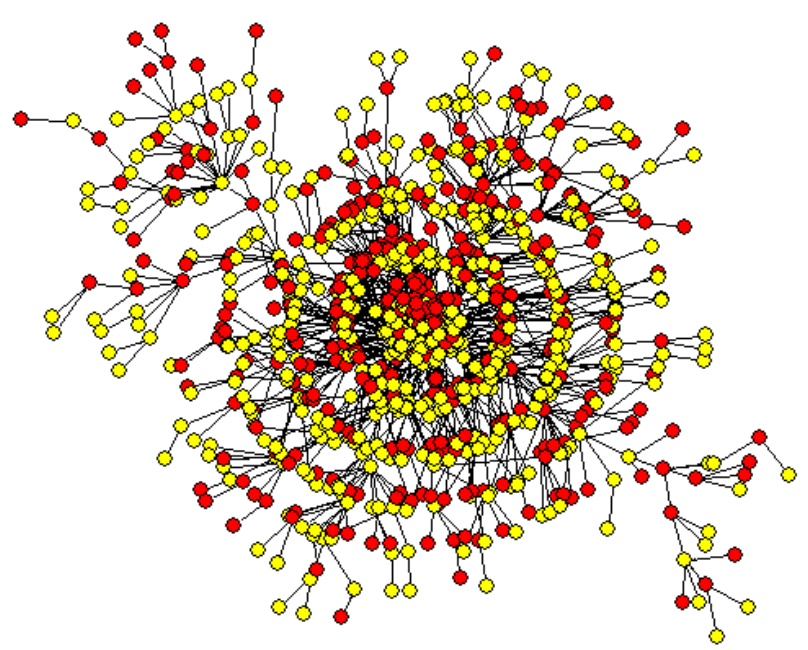

FIG. 1: (Color online) Scale-free tree with spins attached to nodes. Different collors correspond to spin-up (red) and spindown (yellow) orientation when the field reaches $H=0+\delta$. The snap-shot is taken during an avalanche.

domain structure. Compared to the classical Barkhausen avalanches in driven disordered systems [6, 7, 8, 9], apart from the absence of disorder, the following important differences will be pointed out: (i) Only integer values of the local fields $h_{i}$ occur, making the lowest driving rate $\Delta H=1$ finite [19]; (ii) With zero temperature dynamics that we use, back flips are possible due to the antiferromagnetic coupling; (iii) Disconnected avalanches may appear. We consider number of flips before a cascade stops as a measure of the avalanche size $s$. The net magnetization changes may differ from the number of flips during one driving event. We define a time step as one update of the whole network. In the presence of the antiferromagnetic interactions the sequential updates are suitable for all $M$ values. The distributions of avalanche sizes recorded along the ascending branch of hysteresis for the two network topologies and different clustering parameter $M$ are shown in the main Fig. 2

Hysteresis-Loop Criticality. We first discuss in detail the case $M=1$, corresponding to a tree-like structures. As displayed in Fig. 2 the distributions of avalanche sizes exhibit a typical oscillatory behavior superimposed on an underlying power-law dependences, with different slopes in SFN and EXN. The characteristic peaks correspond to collective spin flips [20] on a given depth layer of the tree (cf. Fig 1). In both cases the depth of a graph is proportional to $D \propto \log (N)$ (with different proportionality constants), however, the layers are more densely populated in the EXN than in the SFN. For instance, the number of spins which are directly linked to the spin at 


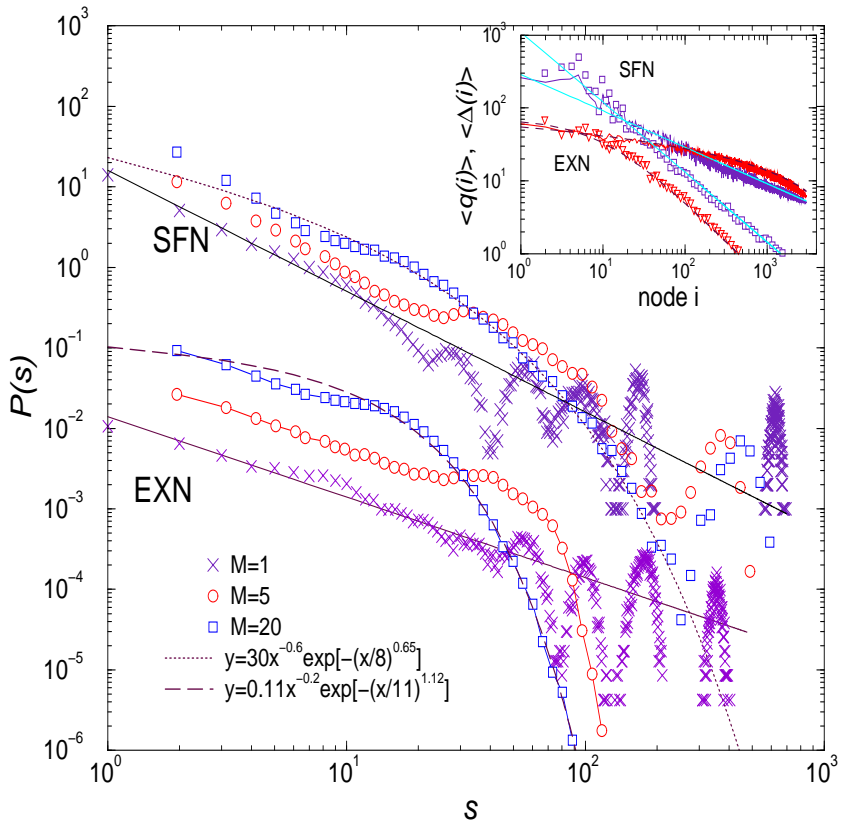

FIG. 2: Probability distribution of avalanche sizes $s$ for different clustering parameter $M=1,5,20$. Two sets of curves correspond to scale-free and exponential networks with $N=10^{3}$ nodes, averaged over $N_{s}=10^{3}$ network realizations. Inset: Profile of the local connectivity $\langle q(i)\rangle$ (full lines) and number of triangles $\Delta(i)$ (symbols) for the two network types, $N=3000, N_{s}=10$. Fits: dashed lines $\langle q(i)\rangle \propto \log \left(10^{4} / i\right)$, $\langle\Delta(i)\rangle=70(1+i / 10)^{-1.12}$; solid lines slopes: -0.5 and -1 .

the hub node (first layer) in SFN is sixfold larger than in the EXN of the same size (cf. inset to Fig. 2). The population differences along layers roughly correspond to the relative positions of peaks in Fig. 2

Typically, spin at the hub is reversed early, then the structure around the hub remains stable until the field reaches very high values. This is compatible with the time picture, (Fig. 3(b) shows $M=5$ case), where the magnetization increase in the SFN takes much longer time, compared with the EXN. A snap shot of the spin-up and spin-down populations corresponding to the middle of the hysteresis loop in SFN is displayed in Fig. 1 Broad network connectivity distribution does not allow definition of magnetic sublattices, such as familiar on Bravais' crystal structures. Moreover, the evolution of the density of domain walls has a characteristic profile for each network structure, as shown in Fig. [3(b). This results in the hysteresis loop of particular properties. Fig. 3(a) show the hysteresis loop for the SFN, which is dominated by the presence of the hub: Both, the extended narrow tails, and the discontinuity jump at the critical field, are attributed to the large connectivity of the hub node.

With increased clustering of networks, i.e., when the parameter $M>1$, closed loops of interacting spins appear. The nearest-neighbor triangles have largest im- pact to the spin dynamics, being incompatible with the antiferromagnetic interactions. This leads to frustration of spins, a familiar concept in the theory of spin-glasses 21]. However, the observed inhomogeneous distribution of triangles on the scale-free graph, as shown in the inset to Fig. 2 affects the hysteresis loop criticality in different manner, compared with the case of spin-glasses [22]. In particular, the critical field $H_{c}$ of the magnetization reversal increases with the clustering on the scale-free network and a finite magnetization discontinuity persists even for very large clustering (see Fig. 31(a)). On the other hand, the absence of the hub nodes in a mild logarithmic profile of the EXN, results in a narrow hysteresis loop and the absence of the large discontinuity for all $M>1$, more similar to the case of Bravais' solids [22].

Increased clustering results in stronger pinning [16]. Consequently, the cut-off decreases in the avalanche size distribution, with gradual smearing of the oscillations. The distributions approach the form that can be fitted by a stretched exponential function for $M=20$. The qualitatively same behavior with different parameters is found in both types of network structures (cf. Fig. 2). The distributions of avalanche durations are broad with increased cut-offs for large clustering. However, owing to small depth ("small-world" feature) of the networks, the overall range of the distributions is short (about one decade) to determine a precise functional form 20].

In order to understand the nature of the hysteresis loop criticality in driven disordered ferromagnets, recently several exact results on Bethe lattice 10 and other sophisticated theoretical approaches were applied 23. It is likely that these approaches, based on the analogy of the Barkhausen avalanches with the stochastic branching processes and bootstrap percolation, can be useful for the study of the hysteresis curves on networks, with a necessary adjustments that will take into account two essentially different points: (i) antiferromagnetic interactions and (ii) power-law connectivity profile. In particular, based on our numerical results in Fig. 2 we expect that true criticality can be proved in the case of tree structures $(M=1)$ with the scaling exponents of the avalanche sizes expressible in terms of the connectivity profile $\gamma$ as $\tau_{s}=1+\gamma=1.5$ for the scale-free, and $\tau_{s}=1$ for the logarithmic profile in the exponential tree.

Conclusions. We have shown that topological constraints due to complex geometry of network and antiferromagnetically coupled spins leads to the avalanchelike magnetization reversal and hysteresis-loop criticality without disorder. The broad distribution of the network connectivity and the presence of the hubs in the scale-free structures leads to unexpected features of the magnetization reversal: increased clustering promotes stronger pinning and shorter avalanches, but at the same time increases the critical field and preserves a finite discontinuity in the magnetization. These features may require renewed theoretical concepts. In practical terms, our re- 


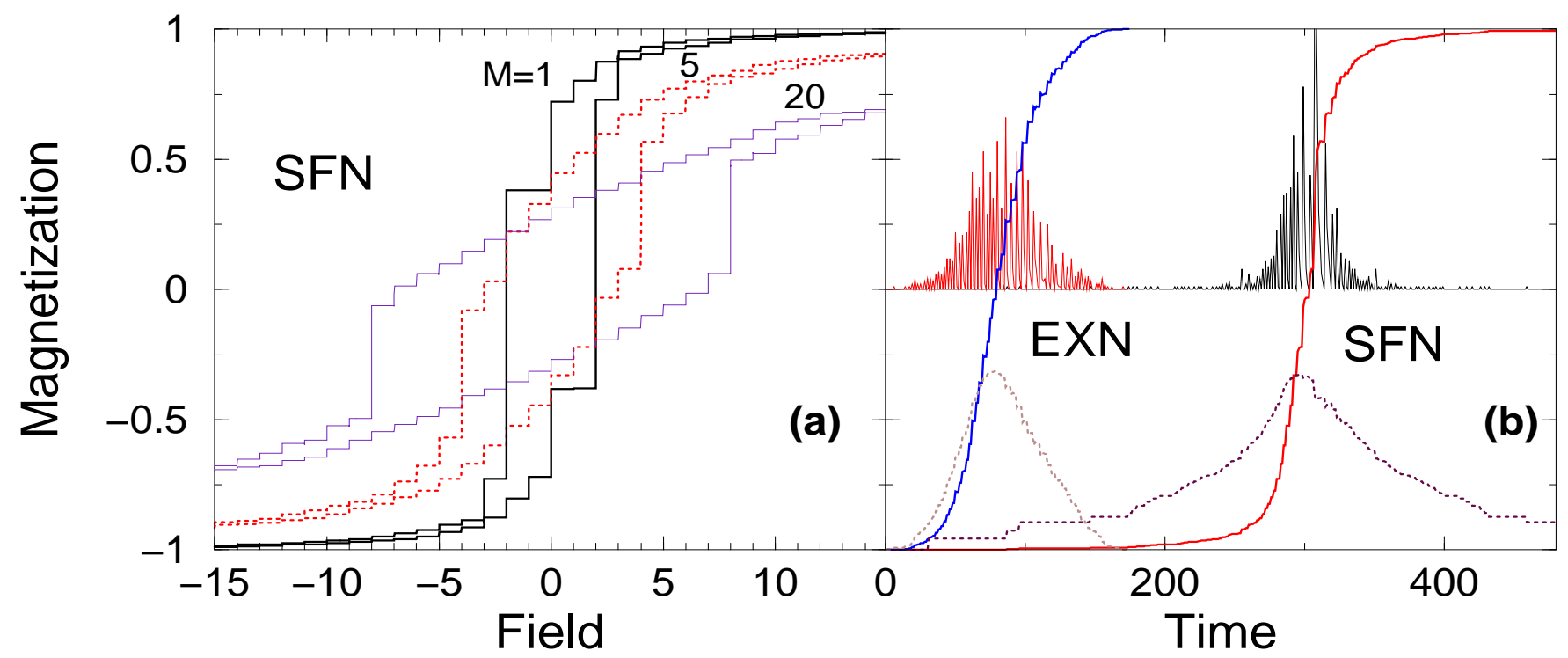

FIG. 3: (a) Hysteresis loop for the scale-free network of different clustering parameter $M=1$ (tree), 5, and 20 . (b) Time dependence of the magnetization, number of flips (Barkhausen noise), and domain-wall density (broken lines, shifted by -1 for better view), for two types of network structures and for $M=5$.

sults suggest that the control of the pattern geometry implies the control of the hysteresis curve properties within an enlarged range of parameters. Our simplified model with "free graphs" may initiate study of more realistic models and practical analysis of integrated nanosystems with rich structural and magnetic characteristics.

B.T. acknowledges support by project No. P1-0044 of the Ministry of Education, Science and Sports (Slovenia). K.M. thanks hospitality at J. Stefan Institute, Ljubljana.

* Electronic address: Bosiljka.Tadic@ijs.si

† URL: http://home.agh.edu.pl/malarz/

‡ Electronic address: kulakowski@novell.ftj.agh.edu.pl

[1] P. Moriarty, Rep. Prog. Phys. 64, 297 (2001).

[2] R.P. Cowburn and M.E. Welland, Science 287, 1466 (2000);

[3] D. Wang et al., Nano Letters, http://pubs.acs.org/cgi-bin/jcen?nalefd/asap/html/nl0345062.hth 1 il. Albert and A.-L. Barabási, Rev. Mod. Phys. 74, 47

[4] S. Gider et al., Appl. Phys. Lett. 69, 3269 (1996); J. Stephens et al., preprint (2004).

[5] S. Miyashita et al., Phys. Rev. Lett. 80, 1525 (1998); U. Nowak et al., Phys. Rev. B 66, 014430 (2002).

[6] For a short review see B. Tadić, Physica A 270, 125 (1999); G. Durin and S. Zapperi, cond-mat/0404512

[7] V. Ya. Shur, Phase Transitions 65, 49 (1998); E. V. Colla, L. K. Chao and M. B. Weissman, Phys. Rev. Lett. 88, 017601 (2002); B. Tadić, Eur. Phys. J. B 28, 81 (2002);

[8] E. Vives et al., Phys. Rev. Lett. 72, 1694 (1994); F. J. Pérez-Reche et al., Phys. Rev. Lett. 87, 195701 (2001).

[9] J. P. Sethna et al., Phys. Rev. Lett. 70, 3347 (1993); E. Vives et al., Phys. Rev. E 52, R5 (1995); B. Tadić, Phys. Rev. Lett. 77, 3843 (1996); Dj. Spasojević et al., Phys. Rev. E 54, 2531 (1996); O. Perkovic et al., Phys. Rev. B 59, 6106 (1999); B. Tadić and U. Nowak, Phys. Rev. E
61, 4610 (2000); E. Vives and A. Planes, Phys. Rev. B 63, 134431 (2001); S. L. A. de Queiroz and M. Bahiana, Phys. Rev. E 64, 066127 (2001); F. J. Pérez-Reche and E. Vives, Phys. Rev. B 67, 134421 (2003).

[10] S. Sabhapandit, P. Shukla, and D. Dhar, J. Stat. Phys. 98, 103 (2000).

[11] A. P. Chernih, V. Ya. Shur, Preprint (in Russian).

[12] M. C. B. Parish and M. Forshaw, Appl. Phys. Lett. 83, 2046 (2003); U. Nowak, R. Chantrell, and E. Kennedy, Phys. Rev. Lett. 84, 163 (2000);

[13] S. Jain and S. Krishna, Proc. Nat. Acad. Sci. 99, 2055 (2002); R. Milo et al., Science 298, 824 (2002); V. P. Zhigulin, Phys. Rev. Lett. 92, 238701 (2004); M. Argollo de Menezes, A.-L. Barabasi, cond-mat/0406421

[14] B. Tadić and S. Thurner, Physica A 332, 566 (2004); Physica A (in press).

[15] S. N. Dorogovtsev and J.F.F. Mendes, Evolution of Networks, Oxford University Press (2003).

[16] D. Stauffer, K. Kulakowski, TASK Quarterly 7, 257 (2003); A. Aleksiejuk, J. Hołyst, and D. Stauffer, Physica A 310, 260 (2002).

[18] B. Tadić, Physica A 293, 273 (2001); 314, 278 (2002).

[19] A general scenario of driving rate effects was discussed in F.-J. Perez-Reche et al., Phys. Rev. Lett. (in press).

[20] Detailed analysis will be given in K. Malarz, J. Karpińska, K. Kułakowski, B. Tadić, (in preparation).

[21] K. H. Fisher and J. A. Hertz, Spin Glasses, Cambridge Univ. Press, (1991); M. B. Weissman, Rev. Mod. Phys., 65, 829 (1993).

[22] In spin-glasses a small concentrations of antiferromagnetic disorder bonds results in a large frustration and slim hysteresis, J. R. Petta, M.B. Weissman, and K. P. O’ Brien, Phys. Rev. E 54, R1029 (1996) .

[23] D. Dhar and P. Shukla, Phys. Rev. Lett. 88, 197202 (2002). 